\title{
Molecular and Functional Alterations in a Mouse Cardiac Model of Friedreich Ataxia
}

\section{Activation of the Integrated Stress Response, eIF2 $\alpha$ Phosphorylation, and the Induction of Downstream Targets}

\author{
Michael Li-Hsuan Huang, ${ }^{*}$ Sutharshani Sivagurunathan, ${ }^{*}$ Samantha Ting, ${ }^{*}$ Patric J. Jansson, ${ }^{*}$ Christopher J.D. Austin, ${ }^{*}$ \\ Matthew Kelly, ${ }^{\dagger \ddagger}$ Christopher Semsarian, ${ }^{\dagger \dagger \S}$ Daohai Zhang, ${ }^{*}$ and Des R. Richardson*
}

From the Molecular Pharmacology and Pathology Program, ${ }^{*}$ Department of Pathology, Sydney Medical School, ${ }^{\ddagger}$ University of Sydney, Sydney; the Agnes Ginges Centre for Molecular Cardiology, Centenary Institute, ${ }^{\dagger}$ Sydney; and the Department of Cardiology, ${ }^{\S}$ Royal Prince Alfred Hospital, Camperdown, Australia

Accepted for publication

May 31, 2013.

Address correspondence to Des R. Richardson, Ph.D., D.Sc., The University of Sydney, D06 Blackburn Building, Sydney, NSW 2031, Australia. E-mail: d.richardson@med.usyd.edu.au.

\begin{abstract}
Friedreich ataxia (FA) is a neurodegenerative and cardiodegenerative disease resulting from marked frataxin deficiency. The condition is characterized by ataxia with fatal cardiomyopathy, but the pathogenic mechanisms are unclear. We investigated the association between gene expression and progressive histopathological and functional changes using the muscle creatine kinase conditional frataxin knockout (KO) mouse; this mouse develops a severe cardiac phenotype that resembles that of FA patients. We examined KO mice from 3 weeks of age, when they are asymptomatic, to 10 weeks of age, when they die of the disease. Positive iron staining was identified in $\mathrm{KO}$ mice from 5 weeks of age, with markedly reduced cardiac function from 6 weeks. We identified an early and marked up-regulation of a gene cohort responsible for stress-induced amino acid biosynthesis and observed markedly increased phosphorylation of eukaryotic translation initiation factor $2 \alpha$ (p-eIF2 $\alpha$ ), an activator of the integrated stress response, in KO mice at 3 weeks of age, relative to wild-type mice. Importantly, the eIF2 $\alpha$-mediated integrated stress response has been previously implicated in heart failure via downstream processes such as autophagy and apoptosis. Indeed, expression of a panel of autophagy and apoptosis markers was enhanced in $\mathrm{KO}$ mice. Thus, the pathogenesis of cardiomyopathy in FA correlates with the early and persistent eIF2 $\alpha$ phosphorylation, which precedes activation of autophagy and apoptosis. (Am J Pathol 2013, 183: 745-757; http://dx.doi.org/10.1016/j.ajpath.2013.05.032)
\end{abstract}

Friedreich ataxia (FA), the most prevalent hereditary neurodegenerative and cardiodegenerative ataxia, is caused by a reduction in frataxin expression. ${ }^{1}$ A wide variety of models have been assessed to examine the pathological processes in FA with respect to the cardiomyopathy and neurological effects observed in the disease. ${ }^{2-7}$ The cardiomyopathy is of significant concern, because it leads to premature death in FA patients. ${ }^{8} \mathrm{~A}$ prominent cardiac pathology in FA is left ventricular (LV) hypertrophy, which develops into a hypokinetic dilated cardiomyopathy characterized by enlarged end-diastolic and end-systolic diameters coupled with reduced cardiac function. ${ }^{8,9}$

Frataxin is a nuclear-encoded mitochondrial protein. ${ }^{1}$ Its down-regulation leads to mitochondrial iron loading and perturbed cellular iron homeostasis. ${ }^{3,10,11}$ This mitochondrial iron accumulation can potentiate further mitochondrial defects from oxidative stress. ${ }^{12}$ Studies using models of $\mathrm{FA}^{4,13,14}$ and other mitochondrial disorders ${ }^{13,15,16}$ have identified up-regulation of several stress-inducible transcripts, which can be activated by the integrated stress response (ISR). ${ }^{17,18}$

The ISR is a highly conserved eukaryotic adaptation to various cellular stresses that results in phosphorylation of

Supported by a Senior Principal Research Fellowship (no. 571123 to D.R.R.), a Project Grant (no. 632698) from the National Health and Medical Research Council (NHMRC) of Australia (D.R.R.), a grant from the Muscular Dystrophy Association-USA (no. 199272 to D.R.R.), and an NHMRC Practitioner Fellowship (no. 571084 to C.S.). 
the $\alpha$ subunit of eukaryotic translation initiation factor 2 $(\mathrm{eIF} 2 \alpha){ }^{17,18}$ The phosphorylated form of eIF2 $\alpha$ (p-eIF2 $\alpha$ ) inhibits general protein synthesis, but selectively activates the translation of stress-inducible transcripts, particularly the activating transcription factor 4 (Atf4). ${ }^{17,18}$ Four mammalian kinases are known to phosphorylate eIF $2 \alpha$ in response to stress stimuli: i) heme-regulated inhibitor (Hri), which is stimulated by heme deficiency; ii) interferon-induced, double-stranded RNA-activated protein kinase (Pkr), which is activated by viral infection; iii) Pkr-like endoplasmic reticulum kinase (Perk), which is triggered by endoplasmic reticulum (ER) stress; and iv) general control nonrepressed 2 protein kinase (Gcn2), which is activated by amino acid deficiency. ${ }^{17,18}$ Significantly, ISR activation plays a role in the development of cardiac dysfunction via its downstream adaptive processes, namely, autophagy ${ }^{19,20}$ and apoptosis. ${ }^{21,22}$

Autophagy is responsible for the recycling of long-lived and damaged organelles by lysosomal degradation. ${ }^{20}$ Although it is often considered to be a protective mechanism, emerging evidence suggests that excessive stimulation of the autophagic pathway can potentiate cardiomyocyte death. $^{20,23,24}$ Apoptosis also plays a significant role in mediating heart failure from cardiomyocyte death. ${ }^{21,22,25}$ Importantly, both processes are associated with FA pathogenesis $^{3,26}$ and both can be induced by ISR activation. ${ }^{19,22}$ The ISR may therefore potentiate the cardiomyopathy of FA.

We investigated the progression of FA cardiomyopathy using the muscle creatine kinase (MCK) conditional frataxin knockout (KO) mouse, which harbors a frataxin deletion in striated muscle (skeletal and heart muscle) and develops a severe cardiomyopathy that closely mimics that of FA patients. $^{2}$ The histopathological, functional, and molecular changes in these mice were characterized and compared with those observed in FA patients. Previous characterization studies of this KO model were focused on the identification of the endpoint phenotype, with very limited assessment of chronological disease development and its molecular consequences. ${ }^{2}$ In the present study, for the first time in a mammalian FA model and using molecular analyses, we identified early ISR activation via eIF $2 \alpha$ phosphorylation, which was followed chronologically by the activation of autophagic and apoptotic markers in $\mathrm{KO}$ mice. The findings highlight the association of the ISR with frataxin deficiency and its potential in mediating the cardiac phenotype.

\section{Materials and Methods}

\section{Transgenic Mice}

The MCK mouse strain was bred from animals provided by Hélène Puccio and Michel Koenig (Louis Pasteur University, Strasbourg, France), who developed the model. ${ }^{2}$ These animals were bred and handled according to a protocol approved by the University of Sydney Animal Ethics
Committee. Genotyping was performed using tail DNA according to standard techniques. ${ }^{2}$

\section{Human Heart Histology}

Heart autopsy specimens from two male and two female FA patients (Supplemental Table S1) were kindly provided by Dr. Arnulf Koeppen (Albany Medical College, Albany, NY). Heart specimens (pre-embedded in paraffin blocks) from four age- and sex-matched control subjects who died from causes unrelated to FA (Supplemental Table S1) were purchased from the National Disease Research Interchange (Philadelphia, PA). For histological analysis, heart specimens from FA patients were embedded in paraffin blocks. Both FA and control hearts were then sectioned and stained with Perls' Prussian blue, Gömöri trichrome, or H\&E for microscopic assessment of iron deposits, fibrosis, and general tissue architecture, respectively. Human tissues were handled according to a protocol approved by the University of Sydney Human Ethics Committee.

\section{Mouse Heart Histology}

KO and WT mouse littermates were euthanized at different time points, from 3 to 10 weeks of age. The hearts were excised, washed in PBS, blotted dry on tissue paper, and fixed in $10 \%$ formalin. For histological analysis, mouse hearts were cut and embedded in paraffin blocks and then sectioned and stained with Perls' Prussian blue, Gömöri trichrome, or H\&E.

\section{Mouse Echocardiography}

Echocardiography was performed on eight littermates (four WT and four KO mice) at 5, 6, 7, 9, and 10 weeks of age. Echocardiography of these mice was performed implementing two-dimensional and M-mode studies, as described previously. ${ }^{27}$ Left ventricular end-diastolic (LVEDD) and end-systolic (LVESD) chamber dimensions and wall thickness were obtained from M-mode tracings based on measurements averaged from three separate cardiac cycles. Left ventricular fractional shortening (FS) was calculated as FS $=($ LVEDD - LVESD)/LVEDD. Heart rate was recorded throughout the study.

\section{Microarray Processing}

Total RNA was isolated from hearts of eight female littermates (two 4-week-old WT, two 4-week-old KO, two 10week-old WT, and two 10-week-old KO mice). Total RNA was isolated using Invitrogen TRIzol reagent (Life Technologies, Carlsbad, CA). First-strand cDNA and biotinlabeled cRNA synthesis was performed and hybridized to the mouse Affymetrix GeneChip 430 version 2.0 (Affymetrix, Santa Clara, CA). ${ }^{3}$ 


\section{Microarray Data Analysis}

A two-phase strategy was used to identify differentially expressed genes. ${ }^{3}$ Genome-wide screening was performed using Affymetrix GeneChip microarrays, and then low-level analysis was performed with Affymetrix GeneChip operating software version 1.3.0, followed by the GC robust multiarray average (GCRMA) method for background correction and quantile-quantile normalization of expression. Tukey's method for multiple pairwise comparisons was applied to acquire foldchange estimations. Tests for significance were calculated and adjusted for multiple comparisons by controlling the false discovery rate at $5 \% .{ }^{28}$ Definitive evidence of differential expression was obtained from RT-PCR and Western blot assessment of samples used for the microarray analysis and at least three other independent samples. Functional annotation of genes was assigned via Gene Ontology ${ }^{29}$ (http://www. geneontology.org, last accessed March 8, 2012), and classifications were obtained through the Database for Annotation, Visualization and Integrated Discovery (DAVID) version 6.7 (http://david.abcc.ncifcrf.gov, last accessed March 8, 2012).

The complete data set has been deposited with the Gene Expression Omnibus (http://ncbi.nlm.nih.gov/geo; accession number GSE31208).

\section{RT-PCR and Western Blot Analysis}

RNA was isolated and RT-PCR was performed by established methods using the primers listed in Table $1 .^{3,10}$
RT-PCR was shown to be semiquantitative by an optimization protocol demonstrating that it was in the log phase of amplification. Densitometry was performed using Quantity One software version 4.6 (Bio-Rad Laboratories, Hercules, $\mathrm{CA}$ ) and was normalized using the relative Gapdh loading control.

Protein isolation and Western blot analysis were performed using established techniques. ${ }^{3}$ The primary antibodies used were against TfR1 (13-6890; Life Technologies), Atf4 (sc-200; Santa Cruz Biotechnology, Santa Cruz, CA), Mthfd2 (ab37840; Abcam, Cambridge, UK), Asns (1732-1; Epitomics, Burlingame, CA), Chop (SC-575; Santa Cruz), p-eIF2 $\alpha$ (Ser51; 3398; Cell Signaling Technology, Danvers, MA), eIF2 $\alpha$ (2103; Cell Signaling Technology), Atg3 (3415; Cell Signaling Technology), LC3 (PD014; MBL International, Woburn, MA), p62 (GP62-C; Progen Biotechnik, Heidelberg, Germany), caspase12 (2202; Cell Signaling Technology), Bax (2772; Cell Signaling Technology), Bcl-2 (2870; Cell Signaling Technology), Itgblbp3 (K0099-3; MBL International), p-Perk (Thr980; 3179; Cell Signaling Technology), Perk (3192; Cell Signaling Technology), Bip (3177; Cell Signaling Technology), Hri (07-728; Millipore, Sydney, Australia), Fech (from Harry Dailey, University of Georgia ${ }^{30}$ ), Fundc1 (ARP53280_P050; Aviva Systems Biology, San Diego, CA), and Gapdh (SC-25778; Santa Cruz Biotechnology). Secondary antibodies used were goat anti-rabbit, rabbit anti-goat, goat anti-mouse, and rabbit anti-chicken (A0545, A5420, A9917, and A9046, respectively; 1/10000; Sigma-Aldrich, St. Louis, MO) conjugated with horseradish peroxidase.

Table 1 Primers for Amplification of Mouse mRNA

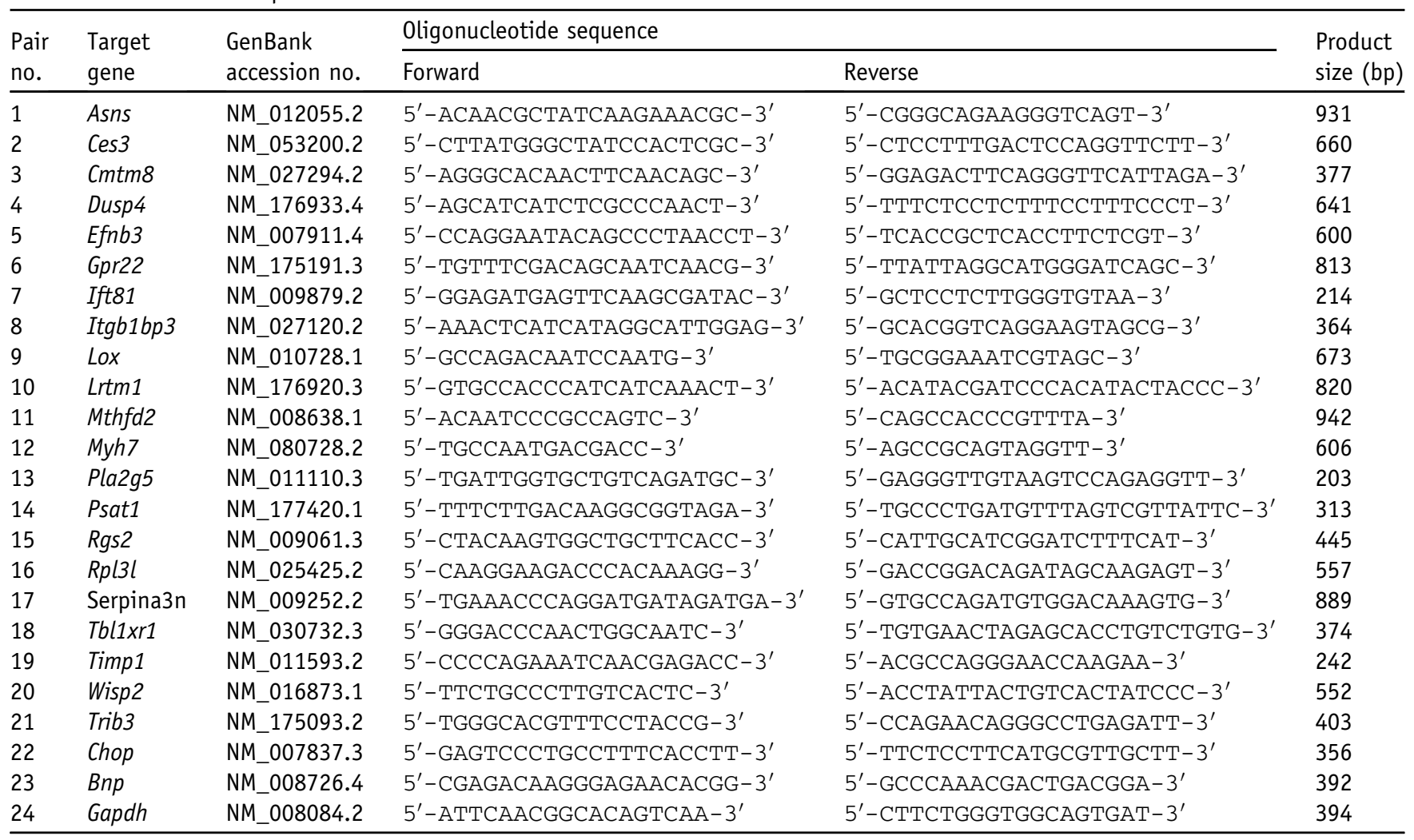




\section{Statistical Analysis}

Data were compared using Student's $t$-test or one-way analysis of variance. Data were considered statistically significant at $P<0.05$. Results are expressed as means \pm SEM.

\section{Results}

\section{MCK Mice Exhibit Severe Cardiac Hypertrophy}

MCK conditional frataxin KO mice develop a cardiomyopathy that is not evident at 4 weeks of age, but becomes pronounced at 10 weeks of age, when the animals die of heart failure. ${ }^{2,3}$ In addition to mitochondrial iron accumulation caused by dysregulated mitochondrial iron metabolism, ${ }^{2,3} \mathrm{KO}$ mice developed prominent cardiac hypertrophy by 10 weeks of age (Figure 1A), as indicated by marked increase in heart-to-body weight ratio as a function of time. ${ }^{2,3}$ This morphological change at this age was associated with the reactivation of the fetal gene program, ${ }^{31}$ as suggested by the significant up-regulation of the hypertrophic markers b-type natriuretic peptide (Bnp) and cardiac myosin heavy chain- $\beta$ (Myh7) $(P<0.01)$ (Figure 1B).

KO and WT mouse hearts were collected between 3 and 10 weeks of age to examine gross structural alterations. We observed no significant differences in $\mathrm{KO}$ mouse hearts from 3 to 5 weeks of age, compared with hearts of agematched WT littermates (Figure 1, C-E and J-L). In contrast, at 6 weeks of age enlargement of the ventricular chambers was observed in hearts of $\mathrm{KO}$ mice, relative to WT littermates (Figure 1, F and M); disease progressively developed into dilation in all cardiac chambers, most prominent in 10-week-old $\mathrm{KO}$ mice, compared with WT littermates (Figure 1, I and P). These features are similar to those observed in human FA patients with late-stage, severe cardiomyopathy. ${ }^{9,32}$

\section{Cardiac Histopathology}

To characterize the histopathological changes in MCK frataxin KO mice with respect to those seen in FA patients (Supplemental Table S1), KO and WT mouse hearts between 5 and 10 weeks were compared with those of FA patients and age- and sex-matched control subjects (Figure 2). In FA patient hearts, the known histopathological features of $\mathrm{FA}^{32}$ were observed (Figure 2, A-F), including cardiomyocyte iron accumulation (Figure 2, A and D), interstitial fibrosis (Figure 2, B and E), and myocyte degeneration, polymorphic cardiomyocytes, and myofiber disarray (Figure 2, C and F).

Histopathological comparisons of hearts were also made between WT mice and KO littermates at onset ( 5 to 7 weeks of age) (Figure 2, G-L) and at end stage (10 weeks of age) (Figure 2, M-R). The WT mouse hearts exhibited no histopathology (Figure 2, G-I and $\mathrm{M}-\mathrm{O}$ ), whereas $\mathrm{KO}$ mouse hearts exhibited classic histopathology associated with FA as early as 5 weeks of age (Figure $2, \mathrm{~J}-\mathrm{L}$ and $\mathrm{P}-\mathrm{R})$. Compared with WT littermates, KO mouse hearts exhibited i) progressive iron accumulation from 5 to 10

A

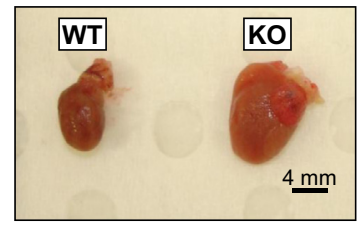

B
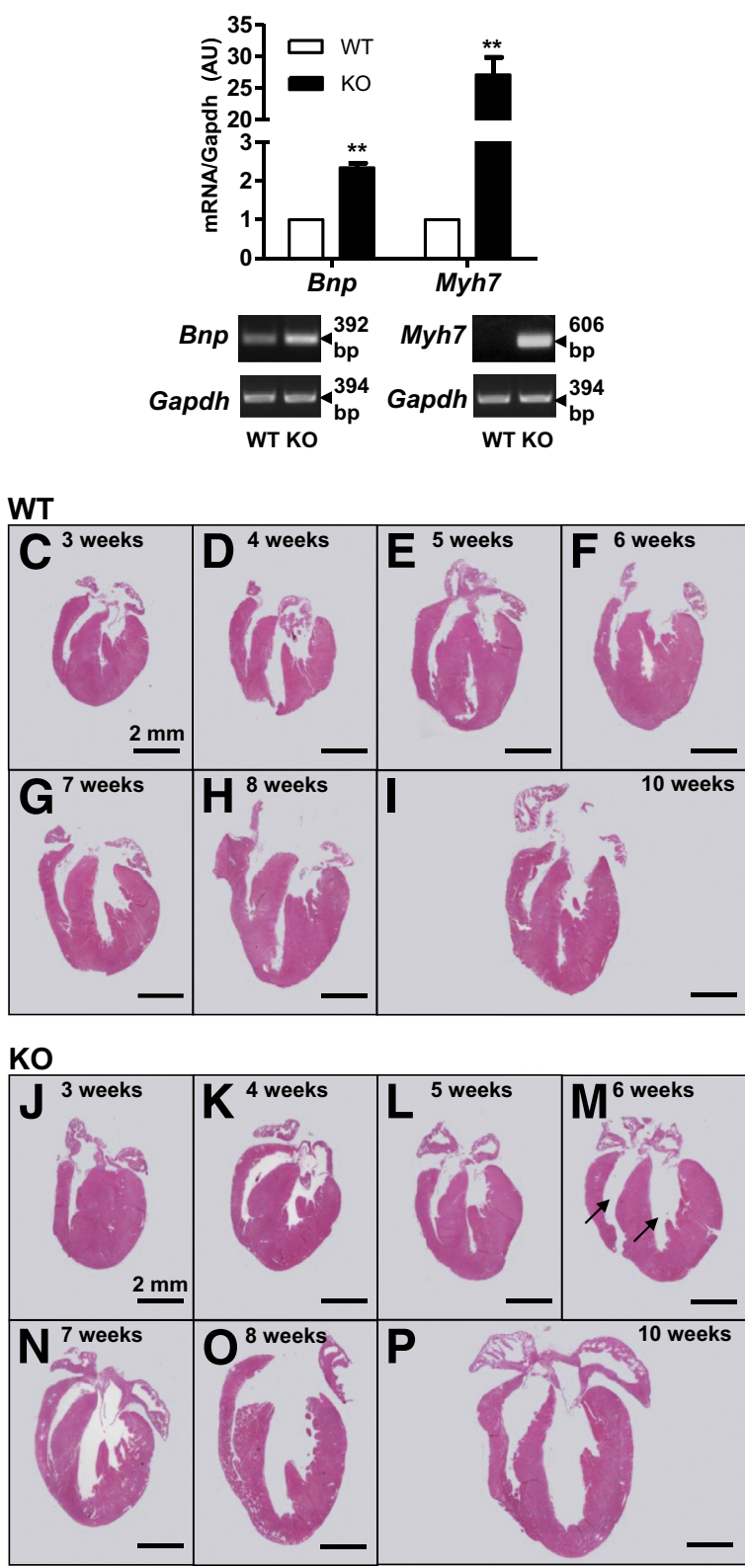

Figure 1 Cardiac phenotype of the MCK conditional frataxin KO mouse. A: Isolated hearts of 10-week-old WT and K0 littermates. B: RT-PCR analysis of the cardiac hypertrophy biomarkers Bnp and Myh7. C-P: Low-power photomicrographs of longitudinal H\&E-stained sections of isolated hearts from 3- to 10-week-old WT (C-I) and KO (J-P) littermates. Images are representative of three or four pairs of littermates per age. Data are expressed as means $\pm \mathrm{SEM}$ and are representative of three or four experiments. ${ }^{* *} P<0.01$. Scale bar $=4 \mathrm{~mm}(\mathbf{A}) ; 2 \mathrm{~mm}(\mathbf{C}-\mathbf{P})$. 

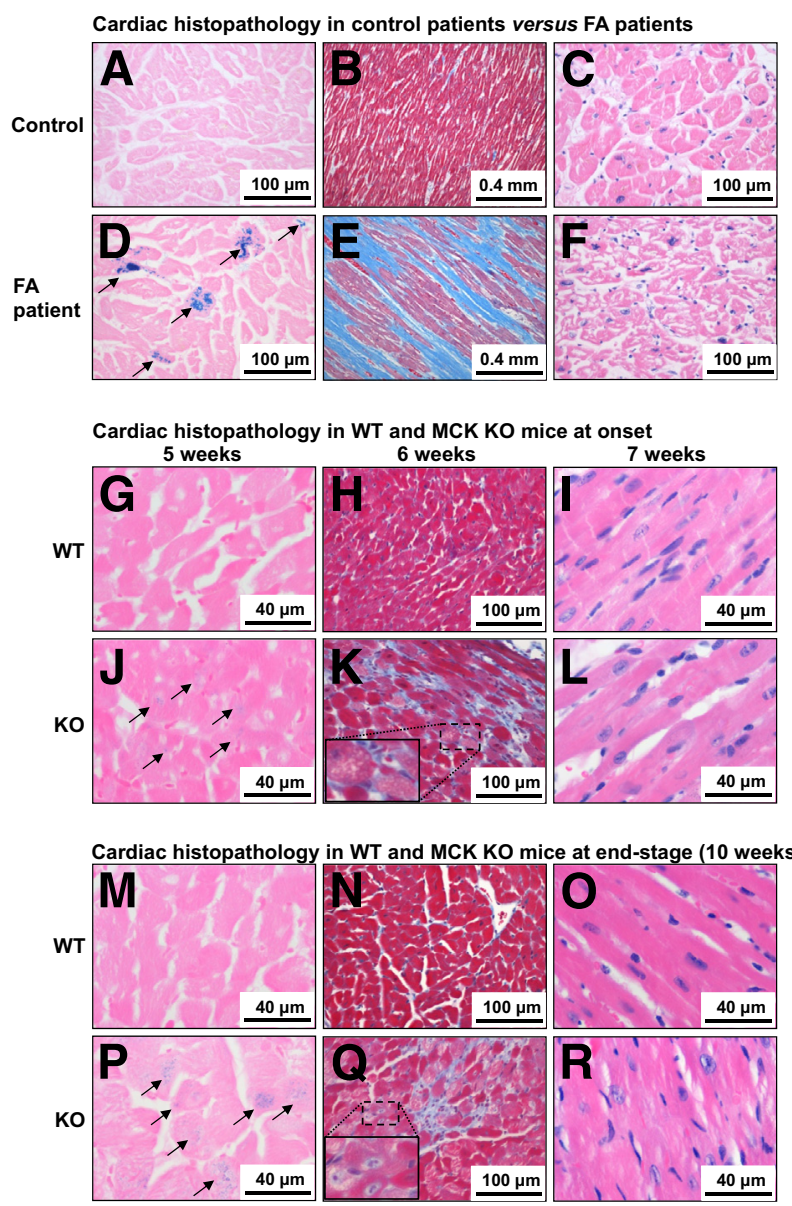

Figure 2 Cardiac histopathology of FA patients and frataxin KO mice, compared with age- and sex-matched control subjects and WT mice, respectively. Histological staining of hearts from age- and sex-matched control subjects $(\mathbf{A}-\mathbf{C})$ and FA patients $(\mathbf{D}-\mathbf{F})$, as well as from 5- to 7week-old (G-L) and 10-week-old (M-R) WT and K0 littermates. In mice, the onset of FA histopathological features can be observed at 5 to 7 weeks of age $(\mathbf{G}-\mathbf{L})$ and continues to 10 weeks of age $(\mathbf{M}-\mathbf{R})$, when $\mathrm{K} 0$ mice die of cardiomyopathy. Hearts were stained with Perls' Prussian blue (left panels) for iron deposits, Gömöri trichrome (middle panels) for fibrosis, and H\&E (right panels) for tissue architecture. Iron-positive cardiomyocytes are indicated by arrows. Blue-gray staining indicates myocardial fibrosis in the FA patient and $\mathrm{KO}$ mice $(\mathbf{E}, \mathbf{K}$, and $\mathbf{Q})$. Insets ( $\mathbf{K}$ and $\mathbf{Q}$ ) show a higher magnification view of the smaller boxed region. Scale bars $(0.4 \mathrm{~mm}, 40 \mu \mathrm{m}$, and $100 \mu \mathrm{m})$ are indicated for each panel. Photomicrographs are representative of four human or mouse heart samples.

weeks of age (Figure 2, G and J, M and P), ii) progressive interstitial fibrosis, polymorphic cardiomyocyte size, pleomorphic nuclei, and cardiomyocyte degeneration, as indicated by cytoplasmic vacuolization, from 6 to 10 weeks of age (Figure 2, H and K, N and Q), and iii) myofiber disarray from 7 weeks of age (Figure 2, I and L), which was also evident at the end stage (Figure 2, O and R).

\section{Echocardiographic Analysis Reveals Decreased Cardiac Function from 6 Weeks of Age}

To characterize functional alteration in $\mathrm{KO}$ heart during the pathogenesis, eight littermates (four WT and four KO) were evaluated by echocardiography ${ }^{27}$ at $5,6,7,9$, and 10 weeks of age. At 5 weeks of age, compared with WT littermates, KO mice were asymptomatic with respect to the echocardiographic parameters LVEDD, LVESD, and FS (Table 2). By 6 weeks of age, however, the cardiac function of $\mathrm{KO}$ mice had rapidly deteriorated, with a significant reduction in FS of KO mice relative to WT littermates $(32 \pm 8 \%$ versus $67 \pm 6 \%$; $P<0.01$ ) (Table 2). This was associated with a significant increase in both LVEDD $(P<0.01)$ and LVESD $(P<$ 0.001 ). This trend continued through 7, 9, and 10 weeks of age, when KO mice developed severe hypokinetic-dilated cardiomyopathy characterized by significantly increased LVEDD $(P<0.05$ or $P<0.01)$ and LVESD $(P<0.05$ or $P<0.01)$ and significantly reduced FS $(P<0.001)$. Importantly, by 10 weeks of age, the FS of KO mice was only $9 \pm 1 \%$, compared with $67 \pm 1 \%$ in their WT littermates (Table 2).

Genes with Greatest Differential Expression Are Linked to Remodeling of the Myocardium and Dysregulation of Amino Acid Synthesis

To identify alterations in transcripts that may be associated with the morphological and functional pathology, we assessed the biological processes most affected by KO of frataxin. We reanalyzed our previously described microarray data for 4and 10-week-old KO and WT littermates, ${ }^{3}$ with a focus on examining transcripts showing significant fold change in gene expression, as well as the greatest fold change, between WT and KO littermates (Supplemental Table S2). Using Gene Ontology annotation, these genes can be categorized into biological processes, including amino acid biosynthesis, cell surface receptor-linked signal transduction, and anatomical structure development (Supplemental Table S2). Confirmation with RT-PCR using appropriate primers (Table 1) revealed that genes participating in amino acid synthesis were significantly up-regulated in 4-week-old, asymptomatic KO mice $(P<0.05)$ (Supplemental Figure S1). Furthermore, at 10 weeks of age, alterations in gene expression in the categories of cell surface receptor-linked signal transduction and anatomical structure development suggest that the myocardium is undergoing signaling-induced anatomical remodeling (Supplemental Table S2 and Supplemental Figure S1). In fact, all of the genes in these two categories are associated with pathological cardiac remodeling and cardiomyopathy [eg, the integrin $\beta 1$-binding protein 3 gene (Itgblbp3)]. ${ }^{15}$

\section{Itgb1bp3 and Genes of Amino Acid Biosynthesis Show the Earliest Alterations}

To chronologically characterize alterations in these biological processes, the expression of genes in each group were evaluated from 3 to 10 weeks of age using RT-PCR (Supplemental Figure S2, A-D). The analysis showed that asparagine synthetase (Asns), mitochondrial NAD-dependent 
Table 2 M-Mode and Two-Dimensional Echocardiographic Analysis of WT and K0 MCK Mice at 5, 6, 7, 9, and 10 Weeks of Age

\begin{tabular}{|c|c|c|c|c|c|c|c|c|c|c|}
\hline \multirow[b]{2}{*}{ Variable } & \multicolumn{2}{|l|}{5 weeks } & \multicolumn{2}{|l|}{6 weeks } & \multicolumn{2}{|l|}{7 weeks } & \multicolumn{2}{|l|}{9 weeks } & \multicolumn{2}{|l|}{10 weeks } \\
\hline & WT & KO & WT & KO & WT & KO & WT & KO & WT & KO \\
\hline $\begin{array}{l}\mathrm{HR} \\
\text { (bpm) }\end{array}$ & $495 \pm 7$ & $492 \pm 8$ & $525 \pm 30$ & $520 \pm 20$ & $562 \pm 28$ & $513 \pm 3$ & $555 \pm 31$ & $495 \pm 13$ & $538 \pm 35$ & $513 \pm 13$ \\
\hline $\begin{array}{l}\text { LVEDD } \\
\quad(\mathrm{mm})\end{array}$ & $2.95 \pm 0.21$ & $3.03 \pm 0.12$ & $2.90 \pm 0.1$ & $3.87 \pm 0.23^{* *}$ & $2.73 \pm 0.15$ & $4.17 \pm 0.49^{*}$ & $2.83 \pm 0.15$ & $4.80 \pm 0.46^{*}$ & $2.90 \pm 0.10$ & $5.60 \pm 0.44^{* *}$ \\
\hline $\begin{array}{l}\text { LVESD } \\
\quad(\mathrm{mm})\end{array}$ & $1.15 \pm 0.07$ & $1.42 \pm 0.25$ & $0.97 \pm 0.15$ & $2.63 \pm 0.15^{* * *}$ & $1.13 \pm 0.06$ & $3.37 \pm 0.49^{*}$ & $1.07 \pm 0.06$ & $4.33 \pm 0.50^{* *}$ & $0.97 \pm 0.06$ & $5.10 \pm 0.44^{* *}$ \\
\hline FS $(\%)$ & $61 \pm 0$ & $53 \pm 7$ & $67 \pm 6$ & $32 \pm 8^{* *}$ & $58 \pm 3$ & $19 \pm 2^{* * *}$ & $62 \pm 3$ & $10 \pm 2^{* * *}$ & $67 \pm 1$ & $9 \pm 1^{* * *}$ \\
\hline
\end{tabular}

Data are expressed as means \pm SEM. $n=4$ mice per group. Analysis of variance was used to determine significance.

$* P<0.05$

$* * P<0.01$

$* * * P<0.001$.

methylene-tetrahydrofolate dehydrogenase-methenyltetrahydrofolate cyclohydrolase (Mthfd2), and phosphoserine aminotransferase-1 (Psatl) genes belong to the amino acid biosynthesis functional group, and Itgblbp3, which is categorized in the anatomical structure development and cell surface receptor-linked signal transduction functional groups, displayed differential gene expression between $\mathrm{KO}$ and WT littermates from 3 weeks of age onward. Notably, Itgblbp 3 was the most-induced transcript identified from the microarray analysis (Supplemental Table S2) and the subsequent RT-PCR analysis (Supplemental Figure S2, B and C). Moreover, Itgb1bp3 protein expression was significantly induced from 6 weeks of age $(P<0.001)$ (Supplemental Figure S2E), which coincided with the onset of myocardial enlargement (Figure 1M) and dysfunction (Table 2), consistent with its role in cardiac remodeling and cardiomyopathy. ${ }^{15}$

\section{Early Changes in Amino Acid Biosynthesis Indicate Activation of the ISR}

The significant up-regulation of Asns, Mthfd2, and Psat1 from 3 weeks in KO mice, compared with WT $(P<0.05)$ (Figure 3) strongly suggests an early alteration in amino acid synthesis. Notably, these genes are activated by Atf 4 in response to several cellular stress stimuli ${ }^{17,18,33}$ and mitochondrial DNA damage. ${ }^{16}$ Furthermore, closer examination of the microarray analysis also identified a significant increase in mRNA levels of CCAAT/enhancer-binding protein homologous protein (Chop) and mammalian homolog of Drosophila tribbles (Trib3), as well as a general increase in aminoacyl-tRNA synthetases, at both 4 and 10 weeks of age (Supplemental Table S3), all of which are also known to be induced by Atf4 activation. ${ }^{17,18,33}$ RT-PCR at each time point showed a significant increase in Chop and Trib3 expression from 4 weeks of age in $\mathrm{KO}$ mice, relative to their WT littermates $(P<0.05)$ (Figure 3). Collectively, these results are consistent with Atf4-induced downstream gene expression. ${ }^{17,18,33}$ Indeed, Atf4 protein expression was significantly increased in 3-week-old $\mathrm{KO}$ mice, compared with their WT littermates $(P<0.01)$ (Figure 4A, inset). However, from 4 weeks of age, Atf 4 protein expression was significantly reduced in $\mathrm{KO}$ heart, compared with WT littermates (variously $P<0.05, P<0.01$, or $P<0.001$ ) (Figure 4A, inset). The subsequent decrease in expression may be attributed to the initial transient induction of Atf4 by stress, followed by the known feedback attenuation of Atf4 activity by downstream molecules. ${ }^{18}$ The reason for the progressive up-regulation of Atf4-inducible genes with age (eg, Mthfd2 in Figure 3), despite attenuation of Atf4 expression from 4 weeks of age, remains unclear. Other transcription factors may be involved in regulating this process. $^{18}$

Western blot analyses also revealed significant and progressive up-regulation of Asns, Mthfd2, and Chop protein in KO heart, compared with the WT heart, from as early as 3 weeks of age (Mthfd2) or 5 weeks of age (Asns and Chop) (variously $P<0.05, P<0.01$, or $P<0.001$ )
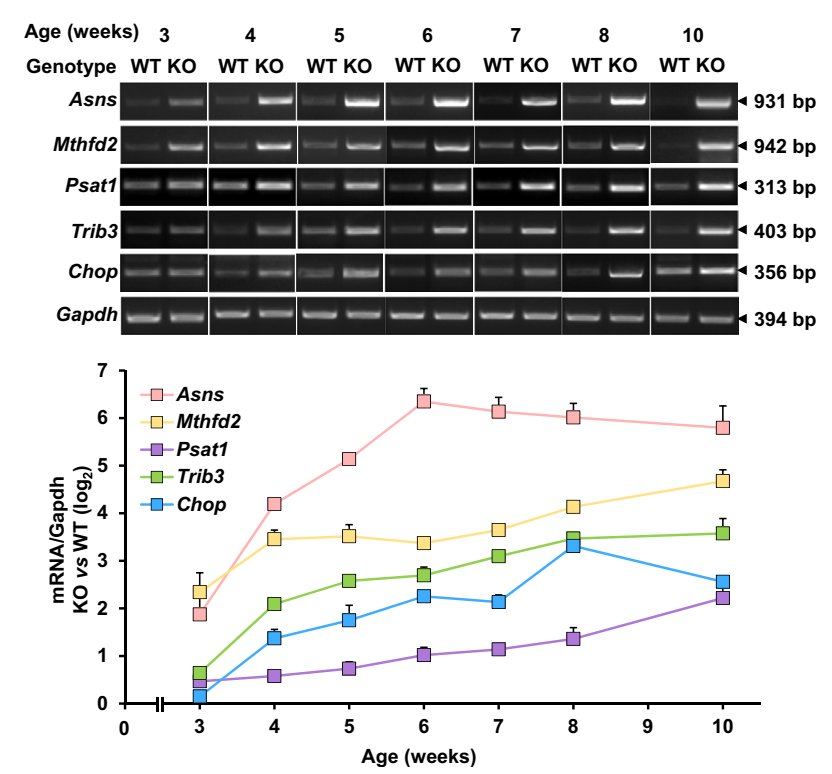

Figure 3 RT-PCR analysis of Atf4-inducible genes suggests activation by Atf4. RT-PCR analysis of Asns, Mthfd2, Psat1, Trib3, and Chop genes in 3- to 10-week-old WT and KO littermates. RT-PCR results shown are representative of three experiments. Gapdh was used as a loading control and implemented for normalization of gene expression. Data are expressed as means \pm SEM and are representative of three experiments. 

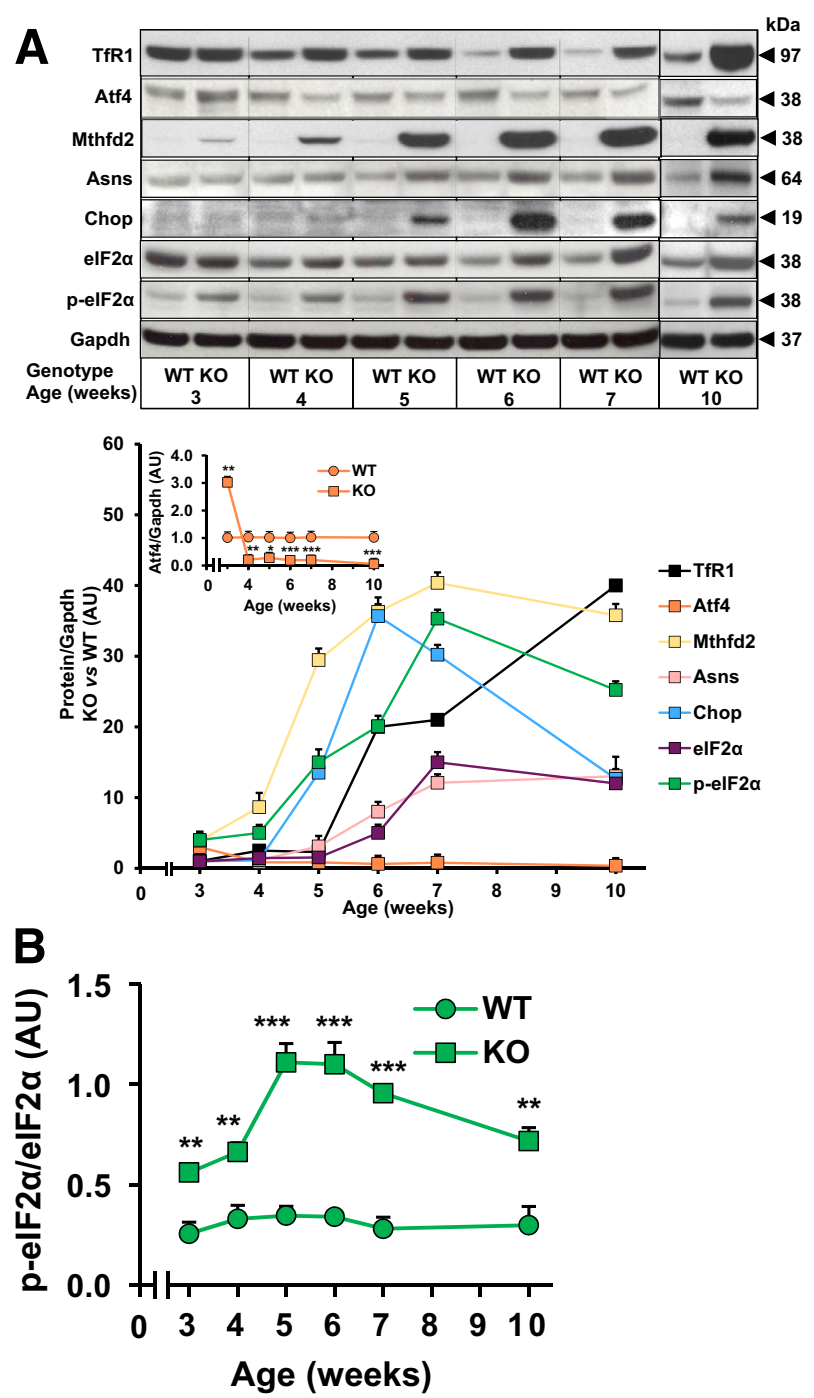

Figure 4 Western blot analysis of ISR-related protein expression over time. A: Western blot analysis of TfR1, Atf4, Mthfd2, Asns, Chop, total eIF2 $\alpha$, and p-eIF2 $\alpha$ expression in 3- to 10-week-old WT and K0 littermates. Gapdh was used as a loading control and implemented for normalization of protein expression. Densitometry data were graphed for protein expression (KO versus WT) and, in the inset, for Atf4/Gapdh expression. Because of well number constraints, samples for 3- to 7-week-old and for 10-week-old mice were run on separate gels but with identical exposure and development conditions. B: Relative phosphorylation of eIF2 $\alpha$ (p-eIF2 $\alpha /$ IF2 $\alpha$ ratio) in WT and KO littermates. Data are expressed as means \pm SEM; data and blots are representative of three to five experiments. ${ }^{*} P<0.05,{ }^{*} P<$ 0.01 , and ${ }^{* * *} P<0.001$.

(Figure 4A). At 3 weeks of age, the induction of Mthfd2 protein precedes up-regulation of the iron transport protein transferrin receptor 1 (TfR1) in KO versus WT mice (Figure 4A). Given that perturbations in TfR1 expression and iron metabolism are known to be the cause of mitochondrial iron accumulation in this KO mouse model, $3,10,11$ the present finding suggests that the activation of the Atf 4 pathway precedes modulation of intracellular iron levels.

Consistent with the transient up-regulation of Atf $4^{17,18}$ at 3 weeks of age, the phosphorylation of the upstream protein
eIF $2 \alpha$ was markedly increased at this time point in $\mathrm{KO}$ mice, compared with WT littermates (Figure 4A). This resulted in a significant increase in the p-eIF $2 \alpha / \operatorname{eIF} 2 \alpha$ ratio (variously $P<0.01$ or $P<0.001$ ) (Figure 4B). Thus, the initial activation of Atf 4 may be due to eIF $2 \alpha$ phosphorylation by upstream kinases responding to intracellular stress signals. ${ }^{17,18}$ The marked and progressive increase in eIF $2 \alpha$ phosphorylation in $\mathrm{KO}$ mouse heart (Figure 4A) indicates that the intracellular stress that triggered its activation not only persisted, but may have been exacerbated. It is notable that total eIF $2 \alpha$ expression also increased from 4 weeks of age in $\mathrm{KO}$ mouse (Figure 4A). However, the relative phosphorylation of eIF $2 \alpha$ versus total eIF $2 \alpha$ in $\mathrm{KO}$ mice significantly exceeded that of their WT littermates at all ages (variously $P<0.01$ or $P<0.001$ ) (Figure 4B), indicating ISR activation. ${ }^{17}$

\section{The Kinases Responsible for eIF2 $\alpha$ Phosphorylation in Frataxin KO Cardiomyocytes}

Up-regulation of the Atf4 transcript was previously identified in a cellular RNAi model of FA and was suggested to be due to ER stress; however, Atf4 protein expression and the phosphorylation status of eIF $2 \alpha$ and its upstream kinases were not examined. ${ }^{14}$ To definitively assess ISR activation by ER stress, we examined the phosphorylation of Perk, the eIF2 $\alpha$ kinase responding to the ER stress stimulus. ${ }^{17}$

No phosphorylation of Perk could be detected by Western blot analysis despite extensive attempts (data not shown), and only very low levels of total Perk protein were observed, which did not differ significantly between WT and KO samples at any time point (Supplemental Figure S3A). However, the expression of the ER stress-induced chaperone binding immunoglobulin protein (Bip) was significantly up-regulated from 6 weeks of age $(P<0.05)$ (Supplemental Figure S3A). Given that Bip is transcriptionally regulated by ER stress, ${ }^{34}$ this marked up-regulation suggests that ER stress may further the activation of the ISR, but is unlikely to be the instigating signal before 3 weeks of age.

In addition to Perk, eIF2 $\alpha$ phosphorylation can be achieved by Gcn2, Pkr, and Hri, which are activated by amino acid deficiency, viral infection, and heme deficiency, respectively. ${ }^{17,18}$ Of these three stimuli, the stimulation of the Hri axis by heme deficiency is a probable cause for eIF $2 \alpha$ phosphorylation in frataxin-deficient cardiomyocytes. This was deduced from the well-characterized perturbation in heme synthesis in KO mice, compared with WT littermates at 4 and 10 weeks of age, ${ }^{3}$ namely, marked downregulation of key enzymes of the heme synthesis pathway, including ferrochelatase (Fech), the final enzyme of this pathway, and a corresponding reduction in myocardial heme level. ${ }^{3}$ In fact, at 3 weeks of age Fech protein expression in KO mice was less than $20 \%$ of the expression in their WT littermates (Supplemental Figure S3A). Furthermore, by 5 weeks of age, Fech expression in KO mice was almost 
ablated. In accord with these observations, the p-Hri/Hri ratio in KO mice, compared with WT littermates, was significantly up-regulated at 3 and 4 weeks of age and then decreased (variously $P<0.05$ or $P<0.01$ ) (Supplemental Figure S3). Thus, early perturbation in heme synthesis due to markedly decreased Fech expression may be involved as part of the Hri axis in phosphorylating eIF $2 \alpha$.

\section{Stress-Induced Cardiac Autophagy}

Given that the ISR can evoke an autophagic response, ${ }^{19}$ the cytosolic vacuolization observed in the cardiomyocytes of $\mathrm{KO}$ mice (Figure 2K, inset) may be suggestive of autophagic vacuole formation. ${ }^{20,24} \mathrm{We}$ therefore evaluated the involvement of autophagy in the cardiomyopathy of MCK $\mathrm{KO}$ mice by examining the expression of three classical autophagic markers: autophagy-related protein Atg3, light chain-3 (LC3), and p62. ${ }^{20,35}$

Atg3, an important early-stage autophagic protein and the E2-like enzyme responsible for conversion of LC3-I to LC3-II, ${ }^{20}$ was significantly up-regulated in KO mice from 3 weeks of age $(P<0.05)$ (Figure 5A), indicating that the autophagic initiation machinery is more active in hearts of KO mice, compared with WT littermates. The conversion of LC3-I to LC3-II during autophagy serves as an autophagy-specific marker. ${ }^{35}$ Western blot analysis revealed a significant loss of LC3-I (18 kDa) from 3 weeks of age in KO mice, relative to WT mice $(P<0.001)$, with a progressive increase in LC3-II $(16 \mathrm{kDa})$ in $\mathrm{KO}$ animals from 6 weeks of age (Figure 5A). This finding led to a significant increase in the LC3-II/LC3-I ratio in KO mice, compared with the WT mice from 6 to 10 weeks of age (variously $P<0.01$ or $P<0.001$ ) (Figure 5A, inset). Moreover, p62 is an essential autophagy substrate that binds and prepares ubiquitinated proteins and organelles for autophagic degradation through its interaction with LC3-II, ${ }^{35}$ and its expression was markedly and significantly up-regulated in $\mathrm{KO}$ mice from 4 weeks onward $(P<0.05)$ (Figure 5A). Considering the dysfunctional mitochondria evident in MCK KO mice, ${ }^{2,3,10,11}$ we assessed the possibility of selective autophagy of the mitochondrion (ie, mitophagy). ${ }^{36}$ Funde1 is a mitochondrial protein that contains a LC3-interacting region to facilitate the selective autophagic degradation of the mitochondrion. ${ }^{36}$ Fundc1 protein was significantly up-regulated in the hearts of the MCK KO mice, compared with WT mice, from 5 weeks of age $(P<0.05)$ (Supplemental Figure $\mathrm{S} 4)$, suggesting that mitophagy was increased in $\mathrm{KO}$ heart.

\section{Apoptosis Is Evident in the Heart of MCK KO Mice}

Chronic ISR activation in the heart leads to apoptosis. ${ }^{21,22}$ Given the late-stage involvement of ER stress, as indicated by Bip expression (Supplemental Figure S3A), we evaluated the cleavage of caspase-12, because this protein plays a crucial role in mitochondrial- and death receptorindependent apoptosis after ER stress. ${ }^{37,38}$ A gradual decrease in the uncleaved form of caspase-12 in KO mice, with a marked and significant up-regulation of its cleaved form in $\mathrm{KO}$ mice, relative to WT littermates, was observed
A
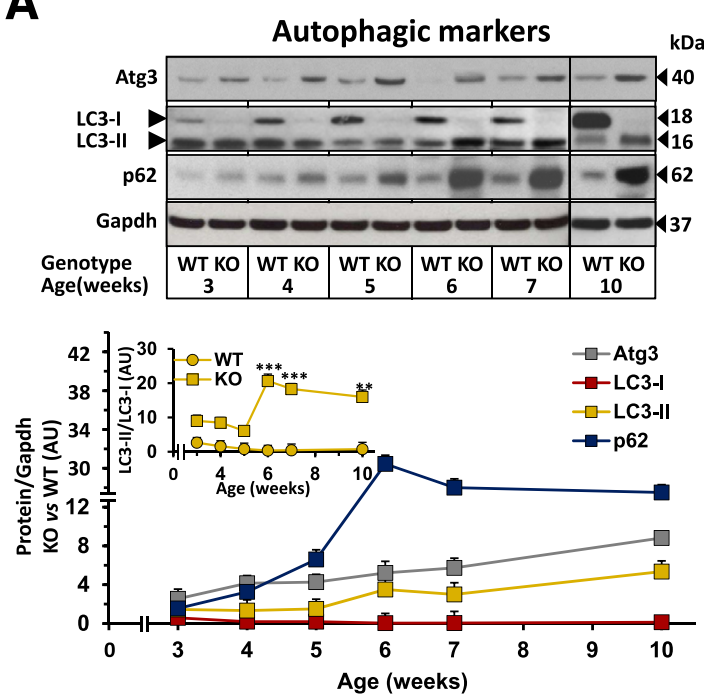

B

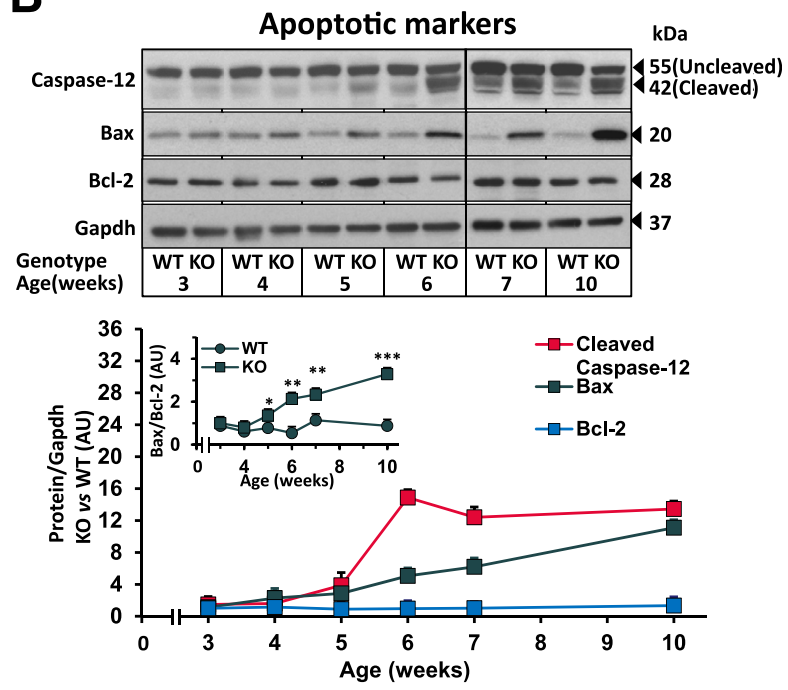

Figure 5 Western blot and densitometry analyses of autophagic (A) and apoptotic (B) markers over time. A: Western blot analysis of Atg3, LC3-I, LC3-II, and p62 expression in 3- to 10-week-old WT and K0 littermates. Gapdh was used as a loading control and implemented for normalization of protein expression. Densitometry data were graphed for protein/Gapdh expression (KO versus WT) and, in the inset, for LC3-II/LC3-I expression. B: Western blot analysis of caspase-12, Bax, and Bcl-2 expression in 3- to 10-week-old WT and K0 littermates. Gapdh was used as a loading control and implemented for normalization of protein expression. Densitometry data were graphed for protein/Gapdh expression (KO versus WT) and, in the inset, for Bax/Bcl-2 expression. Because of well number constraints, samples for 3- to 7-week-old and for 10-week-old mice (A) and for 3- to 6-week-old and for 7- and 10-week-old mice (B) were run on separate gels, but with identical exposure and development conditions. Data are expressed as means $\pm \mathrm{SEM}$; data and blots are representative of three or four experiments. ${ }^{*} P<0.05,{ }^{* *} P<0.01$, and ${ }^{* * *} P<0.001$. 
from 6 weeks of age $(P<0.001)$ (Figure 5B). These results indicate that ER stress might play a role in the induction of apoptosis in the later stages of KO cardiomyopathy.

The process of apoptotic cell death involves a delicate balance between pro- and antiapoptotic proteins. ${ }^{25}$ Thus, to assess the involvement of ISR-induced apoptosis in KO mice, we evaluated the expression of the antiapoptotic protein Bcl-2, relative to Bax (a proapoptotic member of the Bcl-2 protein family ${ }^{25}$ ). Although Bcl-2 expression was not significantly altered between 3 and 10 weeks of age, the levels of Bax were marginally and progressively increased from 3 weeks of age, and become significantly up-regulated from 5 weeks in $\mathrm{KO}$ mice $(P<0.05)$ (Figure 5B). Thus, the marked and significant increase in the relative expression of Bax and Bcl-2 from 5 weeks of age (variously $P<0.05, P<$ 0.01 , or $P<0.001$ ) (Figure 5B, inset) indicated an activation of the apoptotic signal in the MCK KO mouse heart.

\section{Discussion}

With the present study, we have identified changes in gene expression, morphology, and histopathology at key time points during the progression of $\mathrm{KO}$ mouse cardiomyopathy (Figure 6). Histological comparisons between hearts of KO mice and human FA patients confirmed that KO mice exhibit classic FA cardiac histopathology, including cardiomyocyte degeneration, iron deposits, and interstitial fibrosis ${ }^{2,32}$ (Figure 2). Notably, the emergence of iron-positive Perls' Prussian blue staining in 5-week-old KO mouse hearts appeared to be the first observable histopathological feature (Figure 2, G and J), contrary to earlier observations by others using this model. ${ }^{2}$ Longitudinal assessment revealed that, although the iron staining was faint and nonuniform among cardiomyocytes at onset, it became more prominent at later stages, but remained nonubiquitous even at the end stage of the cardiomyopathy (Figure 2, M and P). These observations suggest that perturbations in iron metabolism alone may not be sufficient to account for the pathogenesis of FA cardiomyopathy, which is consistent with findings from a previous study of FA patients. ${ }^{39}$

In the KO mice, the onset of cardiomyocyte degeneration and interstitial fibrosis was first observed at 6 weeks of age (Figure 2K). A causal link between these pathological features and the reduced systolic function associated with the hypokinetic-dilated cardiomyopathy has been suggested in FA patients. ${ }^{9}$ Consistent with this concept, the onset of interstitial fibrosis in MCK KO mice (Figure 2K) coincided with echocardiographic abnormalities, including significant LV dilation and systolic dysfunction (Table 2). Our studies thus demonstrate that, in this FA cardiac mouse model, 5 to 6 weeks marks the transition into a decompensated heart, when progressive histopathological (Figure 2, J-K) and functional cardiac defects (Table 2) ensue. It should be noted that the fibrotic infiltration within $\mathrm{KO}$ heart from 6 weeks of age (Figure 2K) may result in an increased

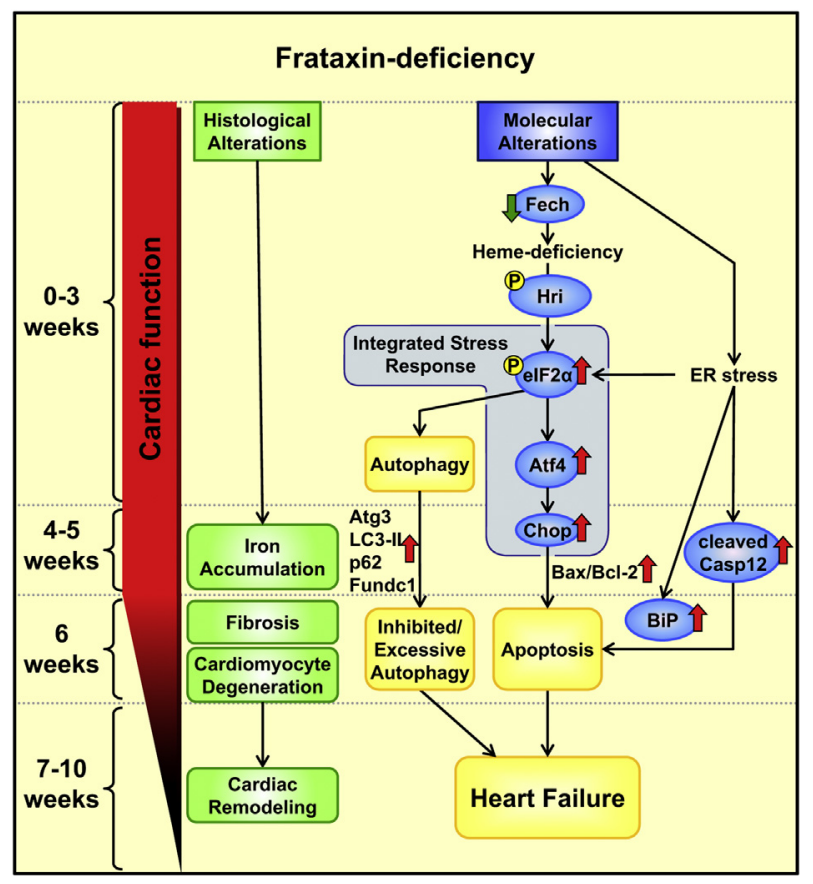

Figure 6 Proposed pathogenesis of heart failure in FA. Frataxin deficiency results in the early activation of the ISR via phosphorylation (P) of eIF2 $\alpha$ and induction of its downstream stress-inducible molecules, including Atf4 and Chop. The precise stress stimulus for the ISR activation is unclear, but may involve early and pronounced perturbation in heme synthesis (markedly reduced Fech expression) acting through Hri in $\mathrm{KO}$ mice, compared to WT mice. In addition, the later involvement of ER stress (up-regulation of Bip and cleaved caspase-12) could potentiate the prolonged activation of the ISR pathway. ISR activation subsequently enhances autophagy (upregulation of Atg3, LC3-II, p62, and Fundc1) and apoptosis (increased expression of Chop, Bax and $\mathrm{BCl}-2$, and cleaved caspase-12), leading to cardiomyocyte death. Histological alterations are first apparent from 5 weeks of age with iron accumulation, followed by fibrosis and cardiomyocyte degeneration from 6 weeks of age. Severe progressive reduction in cardiac function ensues, resulting in heart failure. Casp12, caspase-12.

percentage of noncardiomyocytes, which could interfere with subsequent molecular analyses. However, the cells of the fibrotic repair process are normal and are not undergoing significant apoptosis or autophagy. Consistent with this concept, and in very good correlation with the histopathological observations, we still observed increased autophagy and apoptosis as a function of disease progression (Figure 5). Thus, although the increased contribution of the normal cells involved in the repair process must be acknowledged, the pathological processes observed via histopathology correlate nicely with the molecular alterations shown by Western blot analysis.

Considering the early and pronounced up-regulation of Itgblbp3 in $\mathrm{KO}$ mice, this protein is potentially vital for the remodeling of the frataxin-deficient heart. ${ }^{15}$ Of relevance, marked Itgb1bp3 up-regulation is also evident in mice deficient in heart/muscle-specific mitochondrial superoxide dismutase 2 (Sod2), which develop a severe dilated cardiomyopathy with histopathological and functional defects similar to those of frataxin-deficient mice. ${ }^{15}$ In fact, multiple molecular alterations identified in the Sod2-deficient mice were also found in our 
frataxin KO mice, including Wisp2 and Asns up-regulation and Efnb3 down-regulation (Supplemental Table S2 and Supplemental Figure S1). ${ }^{15}$ Studies in myoblasts demonstrate that Itgb1bp3 modulates the integrin-extracellular matrix protein interaction and promotes myocyte adhesion to fibronectin. ${ }^{40}$ Interestingly, the binding of cardiomyocytes to fibronectin induces a hypertrophic and profibrotic response, which includes up-regulation of Wisp2, Bnp, and $M y h 7^{41}$ Itgb1bp3 may therefore be an integral component in modulating cardiac remodeling in frataxin-deficient cardiomyocytes.

Several Atf4-inducible genes related to amino acid biosynthesis (Asns, Mthfd2, and Psatl) and other processes (Trib3, Chop, and a number of tRNA synthetases) ${ }^{17,18,33}$ were up-regulated as early as 3 to 4 weeks of age in $\mathrm{KO}$ mice (Figure 3 and Supplemental Table S3). These represent the earliest cluster of genes to be markedly up-regulated in the asymptomatic KO mice. The early up-regulation of Asns and $M t h f d 2$ has also been identified in previous animal and cellular models of FA, and is thought to be caused by Atf 4 activation triggered by oxidative stress. ${ }^{4,13,14}$ Thus, the present study is the first to ascertain that the up-regulation of Asns, Mthfd2, Chop, and other Atf4-inducible genes is associated with ISR activation, ie the phosphorylation of eIF $2 \alpha$ and induction of Atf4 expression were evident in 3week-old KO mice (Figure 4). Moreover, for the first time, alterations in Trib3 and Chop expression, both of which are intrinsic to cardiac proapoptotic signaling, ${ }^{21,22,42}$ were identified in this FA cardiac model. The progressive induction of the expression of these molecules and their consequential effects represent an unexplored pathogenic pathway in FA.

Chop has stress-induced, proapoptotic transcriptional activity, ${ }^{38}$ and its up-regulation is observed in failing human and mouse hearts. ${ }^{21,22}$ Moreover, Chop is crucial in the development of cardiomyopathies, because pressure overload-induced hypertrophy, fibrosis, and cardiac dysfunction are significantly attenuated in Chop-deficient mice. ${ }^{21}$ Consistently, the onset of these pathological features in the frataxin-deficient heart coincided with marked up-regulation of the Chop protein. Furthermore, Chop has also been implicated in the pathophysiology of diabetes mellitus, ${ }^{43}$ which is known to affect a subset of FA patients. ${ }^{32}$

In failing human and mouse hearts, ER stress activates the ISR and leads to Chop-mediated apoptosis. ${ }^{21,22}$ Similarly, the ER stress response has been suggested in a cellular FA model $^{14}$ and in other mitochondrial disorders due to mitochondrial inhibition. ${ }^{13,16}$ In accord, the expression of ER stress markers, such as Bip ${ }^{34}$ and cleaved caspase- $12,{ }^{37}$ was markedly increased in $\mathrm{KO}$ mice, but only in the later stages of cardiomyopathy (Figures 5B and 6 and Supplemental Figure S3A). Alternatively, Hri, the eIF2 $\alpha$ kinase activated by intracellular heme deficiency, ${ }^{18}$ represents a plausible instigator of the early ISR activation observed in the frataxindeficient heart (Figure 6). Notably, frataxin interacts with Fech, the final enzyme in the heme synthesis pathway, ${ }^{44}$ and our previous studies using MCK KO mice demonstrated that frataxin deficiency results in pronounced down-regulation of five heme synthesis enzymes and so leads to decreased heme levels. ${ }^{3}$ Moreover, severe and progressive depression of Fech expression was observed in our $\mathrm{KO}$ mice from as early as 3 weeks of age, concomitant with increased Hri phosphorylation (Supplemental Figure S3B). However, considering the subsequent Hri dephosphorylation from 5 weeks of age despite severe Fech and heme deficiency in $\mathrm{KO}$ mice, ${ }^{3}$ further investigation would be needed to establish the role of Hri in FA cardiomyopathy.

Progressive and persistent up-regulation of molecules involved in amino acid biosynthesis (ie, Asns, Mthfd2, and Psat1) was observed in MCK KO mice (Figures 3 and 4A and Supplemental Tables S2 and S3), without evidence of the well-described physiological feedback attenuation. ${ }^{18}$ This may also indicate that frataxin-deficient cardiomyocytes are amino acid deprived, leading to the phosphorylation of eIF $2 \alpha$ by Gcn $2 .^{18}$ The enzymes involved in gluconeogenesis are markedly up-regulated in the heart in this $\mathrm{KO}$ model, ${ }^{45}$ strongly suggesting amino acid catabolism. Moreover, frataxin deficiency in vitro leads to perturbations in the sulfur amino acid pathway that involves Mthfd2. ${ }^{46}$ Thus, progressive induction of Mthfd2 expression from 3 weeks of age (Figures 3 and 4) could reflect sulfur amino acid deprivation in frataxin-deficient cardiomyocytes. Given these findings, ISR activation by amino acid deprivation represents another possible mechanism involved in the cardiomyopathy. However, exhaustive attempts at Western blot analysis using multiple antibodies and conditions were unsuccessful at detecting Gen2 phosphorylation (data not shown), which is responsible for phosphorylating eIF $2 \alpha .^{18}$

The early activation of the ISR, as observed in the present study, could also have an initial protective effect in response to the mitochondria dysfunction ${ }^{17,47}$ that is observed in this model. ${ }^{2}$ However, ISR activation has been shown to lead to autophagy, ${ }^{19}$ which in the cardiac context plays a role in the development of cardiac failure. ${ }^{20,23,24}$ It is likely that autophagic activation has a protective role, especially in the initial stages of the disease, as indicated by i) the early activation of the autophagic pathway described here, ii) the widely accepted protective role described for autophagy in the development of several neurodegenerative disorders, ${ }^{48,49}$ and iii) the specific protective role described for autophagy in response to frataxin suppression. ${ }^{50-52}$

Notably, the present study demonstrated a concomitant induction of autophagy after ISR activation from 3 weeks of age in $\mathrm{KO}$ mice, as indicated by down-regulation of LC3-I and up-regulation of Atg3 and p62 (Figure 5A). However, the accumulation of LC3-II and p62 from 6 weeks of age in the heart of $\mathrm{KO}$ mice may indicate a perturbation in autophagy. Notably, through interaction with LC3-II, p62 is incorporated into autophagosomes and is degraded during autophagy. ${ }^{35}$ After the initial increase of LC3-II and p62 in $\mathrm{KO}$ mice, their expression should therefore decrease if the autophagic process comes to completion. Thus, the present results may suggest a decrease in autophagic flux in the heart 
of $\mathrm{KO}$ mice, given the activated autophagic initiation machinery (shown by increased Atg3 and decreased LC3-I) and an inhibition of lysosomal-mediated autophagic degradation (deduced from the accumulation of LC3-II and p62), and/or a sustained and marked increased in the autophagic pathway after 6 weeks, because both LC 3 and p62 can be transcriptionally up-regulated during autophagy. ${ }^{35}$ Both possibilities could indicate a decompensation of the autophagy-mediated survival machinery, resulting in autophagic cell death in cardiomyocytes and subsequent contractile dysfunction. ${ }^{20,23,24}$ Consistent with such observations, our echocardiographic analysis demonstrated a significant reduction in cardiac function in KO mice from 6 weeks of age (Table 2). Thus, the early ISR activation and autophagy may be one of the central pathogenic pathways causing cardiomyopathy in FA (Figure 6). The up-regulation of the mitophagy marker Fundc $1^{36}$ was of interest and is consistent with the marked derangement in mitochondrial metabolism in MCK KO mice. ${ }^{3,10,11}$

Finally, persistent ISR stimulation also leads to apoptosis. ${ }^{22,42}$ In the present study, there was significant upregulation of apoptotic markers in $\mathrm{KO}$ mice from 5 weeks of age, including Bax and cleaved caspase-12 (Figure 5B). The significantly elevated $\mathrm{Bax} / \mathrm{Bcl}-2$ ratio suggests a preference for the execution of cell death through reduced mitochondrial membrane integrity and a subsequent release of cytochrome $c .{ }^{38}$ Moreover, caspase-12, which is localized to the ER membrane, mediates ER stress-specific apoptosis. ${ }^{37,38}$ Thus, taken together with the up-regulation of Bip (Supplemental Figure S3A), these results point to a role for ER stress-induced apoptosis in frataxin-deficient cardiomyocytes (Figure 6).

In summary, with the present study we have characterized for the first time the chronology of histopathological, functional, and molecular alterations in this mouse cardiac model of FA, revealing gene expression changes preceding cardiomyocyte degeneration.

\section{Acknowledgments}

We thank Angelica Merlot and Drs. Katie Dixon, Zaklina Kovacevic, Darius Lane, Vera Richardson, Sumit Sahni, Yohan Suryo Rahmanto, and Yu Yu (University of Sydney) for their assistance in assessing the manuscript.

\section{Supplemental Data}

Supplemental material for this article can be found at http://dx.doi.org/10.1016/j.ajpath.2013.05.032.

\section{References}

1. Campuzano V, Montermini L, Lutz Y, Cova L, Hindelang C, Jiralerspong S, Trottier Y, Kish SJ, Faucheux B, Trouillas P, Authier FJ, Dürr A, Mandel JL, Vescovi A, Pandolfo M, Koenig M:
Frataxin is reduced in Friedreich ataxia patients and is associated with mitochondrial membranes. Hum Mol Genet 1997, 6:1771-1780

2. Puccio H, Simon D, Cossée M, Criqui-Filipe P, Tiziano F, Melki J, Hindelang C, Matyas R, Rustin P, Koenig M: Mouse models for Friedreich ataxia exhibit cardiomyopathy, sensory nerve defect and Fe-S enzyme deficiency followed by intramitochondrial iron deposits. Nat Genet 2001, 27:181-186

3. Huang ML, Becker EM, Whitnall M, Suryo Rahmanto Y, Ponka P, Richardson DR: Elucidation of the mechanism of mitochondrial iron loading in Friedreich's ataxia by analysis of a mouse mutant. Proc Natl Acad Sci USA 2009, 106:16381-16386

4. Seznec H, Simon D, Bouton C, Reutenauer L, Hertzog A, Golik P, Procaccio V, Patel M, Drapier JC, Koenig M, Puccio H: Friedreich ataxia: the oxidative stress paradox. Hum Mol Genet 2005, 14: $463-474$

5. Miranda CJ, Santos MM, Ohshima K, Smith J, Li L, Bunting M, Cossée M, Koenig M, Sequeiros J, Kaplan J, Pandolfo M: Frataxin knockin mouse. FEBS Lett 2002, 512:291-297

6. Coppola G, Choi SH, Santos MM, Miranda CJ, Tentler D, Wexler EM, Pandolfo M, Geschwind DH: Gene expression profiling in frataxin deficient mice: microarray evidence for significant expression changes without detectable neurodegeneration. Neurobiol Dis 2006, 22:302-311

7. Ventura N, Rea SL: Caenorhabditis elegans mitochondrial mutants as an investigative tool to study human neurodegenerative diseases associated with mitochondrial dysfunction. Biotechnol J 2007, 2: 584-595

8. Weidemann F, Rummey C, Bijnens B, Störk S, Jasaityte R, Dhooge J, Baltabaeva A, Sutherland G, Schulz JB, Meier T, Mitochondrial Protection with Idebenone in Cardiac or Neurological Outcome (MICONOS) study group: The heart in Friedreich ataxia: definition of cardiomyopathy, disease severity, and correlation with neurological symptoms. Circulation 2012, 125:1626-1634

9. Casazza F, Morpurgo M: The varying evolution of Friedreich's ataxia cardiomyopathy. Am J Cardiol 1996, 77:895-898

10. Whitnall M, Suryo Rahmanto Y, Sutak R, Xu X, Becker EM, Mikhael MR, Ponka P, Richardson DR: The MCK mouse heart model of Friedreich's ataxia: alterations in iron-regulated proteins and cardiac hypertrophy are limited by iron chelation. Proc Natl Acad Sci USA 2008, 105:9757-9762

11. Whitnall M, Suryo Rahmanto Y, Huang ML, Saletta F, Lok HC, Gutiérrez L, Lázaro FJ, Fleming AJ, St Pierre TG, Mikhael MR, Ponka P, Richardson DR: Identification of nonferritin mitochondrial iron deposits in a mouse model of Friedreich ataxia. Proc Natl Acad Sci USA 2012, 109:20590-20595

12. Huang ML, Lane DJ, Richardson DR: Mitochondrial mayhem: the mitochondrion as a modulator of iron metabolism and its role in disease. Antioxid Redox Signal 2011, 15:3003-3019

13. Cortopassi G, Danielson S, Alemi M, Zhan SS, Tong W, Carelli V, Martinuzzi A, Marzuki S, Majamaa K, Wong A: Mitochondrial disease activates transcripts of the unfolded protein response and cell cycle and inhibits vesicular secretion and oligodendrocyte-specific transcripts. Mitochondrion 2006, 6:161-175

14. Lu C, Cortopassi G: Frataxin knockdown causes loss of cytoplasmic iron-sulfur cluster functions, redox alterations and induction of heme transcripts. Arch Biochem Biophys 2007, 457:111-122

15. Nojiri $H$, Shimizu $T$, Funakoshi $M$, Yamaguchi O, Zhou $H$, Kawakami S, Ohta Y, Sami M, Tachibana T, Ishikawa $\mathrm{H}$, Kurosawa H, Kahn RC, Otsu K, Shirasawa T: Oxidative Stress Causes Heart Failure with Impaired Mitochondrial Respiration. J Biol Chem 2006, 281:33789-33801

16. Fujita Y, Ito M, Nozawa Y, Yoneda M, Oshida Y, Tanaka M: CHOP (C/EBP homologous protein) and ASNS (asparagine synthetase) induction in cybrid cells harboring MELAS and NARP mitochondrial DNA mutations. Mitochondrion 2007, 7:80-88

17. Harding HP, Zhang YH, Zeng HQ, Novoa I, Lu PD, Calfon M, Sadri N, Yun C, Popko B, Paules R, Stojdl DF, Bell JC, Hettmann T, 
Leiden JM, Ron D: An integrated stress response regulates amino acid metabolism and resistance to oxidative stress. Mol Cell 2003, 11: 619-633

18. Kilberg MS, Shan JX, Su N: ATF4-dependent transcription mediates signaling of amino acid limitation. Trends Endocrinol Metab 2009, 20:436-443

19. Kouroku Y, Fujita E, Tanida I, Ueno T, Isoai A, Kumagai H, Ogawa S, Kaufman RJ, Kominami E, Momoi T: ER stress (PERK/ eIF2alpha phosphorylation) mediates the polyglutamine-induced LC3 conversion, an essential step for autophagy formation. Cell Death Differ 2006, 14:230-239

20. Gustafsson $\AA$ B, Gottlieb RA: Autophagy in ischemic heart disease. Circ Res 2009, 104:150-158

21. Fu HY, Okada K, Liao Y, Tsukamoto O, Isomura T, Asai M, Sawada T, Okuda K, Asano Y, Sanada S, Asanuma H, Asakura M, Takashima S, Komuro I, Kitakaze M, Minamino T: Ablation of $\mathrm{C} / \mathrm{EBP}$ homologous protein attenuates endoplasmic reticulummediated apoptosis and cardiac dysfunction induced by pressure overload. Circulation 2010, 122:361-369

22. Okada K, Minamino T, Tsukamoto $\mathrm{Y}$, Liao $\mathrm{Y}$, Tsukamoto $\mathrm{O}$, Takashima S, Hirata A, Fujita M, Nagamachi Y, Nakatani T, Yutani C, Ozawa K, Ogawa S, Tomoike H, Hori M, Kitakaze M: Prolonged endoplasmic reticulum stress in hypertrophic and failing heart after aortic constriction: possible contribution of endoplasmic reticulum stress to cardiac myocyte apoptosis. Circulation 2004, 110: 705-712

23. Knaapen MWM, Davies MJ, De Bie M, Haven AJ, Martinet W, Kockx MM: Apoptotic versus autophagic cell death in heart failure. Cardiovasc Res 2001, 51:304-312

24. Shimomura H, Terasaki F, Hayashi $T$, Kitaura $Y$, Isomura $T$, Suma H: Autophagic degeneration as a possible mechanism of myocardial cell death in dilated cardiomyopathy. Jpn Circ J 2001, 65: 965-968

25. Olivetti G, Abbi R, Quaini F, Kajstura J, Cheng W, Nitahara JA, Quaini E, DiLoreto C, Beltrami CA, Krajewski S, Reed JC, Anversa P: Apoptosis in the failing human heart. N Engl J Med 1997, 336:1131-1141

26. Simon D, Seznec H, Gansmuller A, Carelle N, Weber P, Metzger D, Rustin P, Koenig M, Puccio H: Friedreich ataxia mouse models with progressive cerebellar and sensory ataxia reveal autophagic neurodegeneration in dorsal root ganglia. J Neurosci 2004, 24: $1987-1995$

27. Tsoutsman T, Kelly M, Ng DC, Tan JE, Tu E, Lam L, Bogoyevitch MA, Seidman CE, Seidman JG, Semsarian C: Severe heart failure and early mortality in a double-mutation mouse model of familial hypertrophic cardiomyopathy. Circulation 2008, 117:1820-1831

28. Benjamini Y, Hochberg Y: Controlling the false discovery rate: a practical and powerful approach to multiple testing. J R Stat Soc B Methodol 1995, 57:289-300

29. Ashburner M, Ball CA, Blake JA, Botstein D, Butler H, Cherry JM, Davis AP, Dolinski K, Dwight SS, Eppig JT, Harris MA, Hill DP, Issel-Tarver L, Kasarskis A, Lewis S, Matese JC, Richardson JE, Ringwald M, Rubin GM, Sherlock G, the Gene Ontology Consortium: Gene Ontology: tool for the unification of biology. Nat Genet 2000, 25:25-29

30. Karr SR, Dailey HA: The synthesis of murine ferrochelatase in vitro and in vivo. Biochem J 1988, 254:799-803

31. Oka T, Xu J, Molkentin JD: Re-employment of developmental transcription factors in adult heart disease. Semin Cell Dev Biol 2007, 18:117-131

32. Koeppen AH: Friedreich's ataxia: pathology, pathogenesis, and molecular genetics. J Neurol Sci 2011, 303:1-12

33. Adams CM: Role of the transcription factor ATF4 in the anabolic actions of insulin and the anti-anabolic actions of glucocorticoids. J Biol Chem 2007, 282:16744-16753

34. Gülow K, Bienert D, Haas IG: BiP is feed-back regulated by control of protein translation efficiency. J Cell Sci 2002, 115:2443-2452
35. Mizushima N, Yoshimori T, Levine B: Methods in mammalian autophagy research. Cell 2010, 140:313-326

36. Liu L, Feng D, Chen G, Chen M, Zheng Q, Song P, Ma Q, Zhu C, Wang R, Qi W, Huang L, Xue P, Li B, Wang X, Jin H, Wang J, Yang F, Liu P, Zhu Y, Sui S, Chen Q: Mitochondrial outer-membrane protein FUNDC1 mediates hypoxia-induced mitophagy in mammalian cells. Nat Cell Biol 2012, 14:177-185

37. Nakagawa T, Zhu H, Morishima N, Li E, Xu J, Yankner BA, Yuan J: Caspase-12 mediates endoplasmic-reticulum-specific apoptosis and cytotoxicity by amyloid-beta. Nature 2000, 403:98-103

38. Szegezdi E, Logue SE, Gorman AM, Samali A: Mediators of endoplasmic reticulum stress-induced apoptosis. EMBO Rep 2006, 7: 880-885

39. Michael S, Petrocine SV, Qian J, Lamarche JB, Knutson MD, Garrick MD, Koeppen $\mathrm{AH}$ : Iron and iron-responsive proteins in the cardiomyopathy of Friedreich's ataxia. Cerebellum 2006, 5: 257-267

40. Li J, Rao H, Burkin D, Kaufman SJ, Wu C: The muscle integrin binding protein (MIBP) interacts with alpha7betal integrin and regulates cell adhesion and laminin matrix deposition. Dev Biol 2003, 261:209-219

41. Chen H, Huang XN, Stewart AF, Sepulveda JL: Gene expression changes associated with fibronectin-induced cardiac myocyte hypertrophy. Physiol Genomics 2004, 18:273-283

42. Avery J, Etzion S, DeBosch BJ, Jin X, Lupu TS, Beitinjaneh B, Grand J, Kovacs A, Sambandam N, Muslin AJ: TRB3 function in cardiac endoplasmic reticulum stress. Circ Res 2010, 106:1516-1523

43. Song BB, Scheuner D, Ron D, Pennathur S, Kaufman RJ: Chop deletion reduces oxidative stress, improves beta cell function, and promotes cell survival in multiple mouse models of diabetes. J Clin Invest 2008, 118:3378-3389

44. Yoon T, Cowan JA: Frataxin-mediated iron delivery to ferrochelatase in the final step of heme biosynthesis. J Biol Chem 2004, 279: 25943-25946

45. Sutak R, Xu X, Whitnall M, Kashem MA, Vyoral D, Richardson DR: Proteomic analysis of hearts from frataxin knockout mice: marked rearrangement of energy metabolism, a response to cellular stress and altered expression of proteins involved in cell structure, motility and metabolism. Proteomics 2008, 8:1731-1741

46. Tan G, Napoli E, Taroni F, Cortopassi G: Decreased expression of genes involved in sulfur amino acid metabolism in frataxin-deficient cells. Hum Mol Genet 2003, 12:1699-1711

47. Baker BM, Nargund AM, Sun T, Haynes CM: Protective coupling of mitochondrial function and protein synthesis via the eIF2alpha kinase GCN-2. PLoS Genet 2012, 8:e1002760

48. Harris H, Rubinsztein DC: Control of autophagy as a therapy for neurodegenerative disease. Nat Rev Neurol 2012, 8:108-117

49. Mariño G, Madeo F, Kroemer G: Autophagy for tissue homeostasis and neuroprotection. Curr Opin Cell Biol 2011, 23:198-206

50. Palomo GM, Cerrato T, Gargini R, Diaz-Nido J: Silencing of frataxin gene expression triggers p53-dependent apoptosis in human neuronlike cells. Hum Mol Genet 2011, 20:2807-2822

51. Marobbio CM, Pisano I, Porcelli V, Lasorsa FM, Palmieri L: Rapamycin reduces oxidative stress in frataxin-deficient yeast cells. Mitochondrion 2012, 12:156-161

52. Schiavi A, Torgovnick A, Kell A, Megalou E, Castelein N, Guccini I, Marzocchella L, Gelino S, Hansen M, Malisan F, Condò I, Bei R, Rea SL, Braeckman BP, Tavernarakis N, Testi R, Ventura N: Autophagy induction extends lifespan and reduces lipid content in response to frataxin silencing in C. elegans. Exp Gerontol 2013, 48:191-201

53. Adams JW, Pagel AL, Means CK, Oksenberg D, Armstrong RC, Brown JH: Cardiomyocyte apoptosis induced by Galphaq signaling is mediated by permeability transition pore formation and activation of the mitochondrial death pathway. Circ Res 2000, 87:1180-1187

54. Takimoto E, Koitabashi N, Hsu S, Ketner EA, Zhang M, Nagayama T, Bedja D, Gabrielson KL, Blanton R, Siderovski DP, Mendelsohn ME, Kass DA: Regulator of $G$ protein signaling 2 mediates cardiac 
compensation to pressure overload and antihypertrophic effects of PDE5 inhibition in mice. J Clin Invest 2009, 119:408-420

55. Zhang W, Anger T, Su JL, Hao JM, Xu XM, Zhu M, Gach A, Cui L, Liao RL, Mende U: Selective loss of fine tuning of Gq/11 signaling by RGS2 protein exacerbates cardiomyocyte hypertrophy. J Biol Chem 2006, 281:5811-5820

56. Tsuda T, Gao E, Evangelisti L, Markova D, Ma X, Chu ML: Postischemic myocardial fibrosis occurs independent of hemodynamic changes. Cardiovasc Res 2003, 59:926-933

57. Sivakumar P, Gupta S, Sarkar S, Sen S: Upregulation of lysyl oxidase and MMPs during cardiac remodeling in human dilated cardiomyopathy. Mol Cell Biochem 2008, 307:159-167

58. Funaba M, Zimmerman CM, Mathews LS: Modulation of Smad2mediated signaling by extracellular signal-regulated kinase. J Biol Chem 2002, 277:41361-41368

59. Kurzepa J, Szczepanska-Szerej A, Stryjecka-Zimmer M, MaleckaMassalska T, Stelmasiak Z: Simvastatin could prevent increase of the serum MMP-9/TIMP-1 ratio in acute ischaemic stroke. Folia Biol (Praha) 2006, 52:181-183
60. Lubos E, Schnabel R, Rupprecht HJ, Bickel C, Messow CM, Prigge S, Cambien F, Tiret L, Munzel T, Blankenberg S: Prognostic value of tissue inhibitor of metalloproteinase-1 for cardiovascular death among patients with cardiovascular disease: results from the AtheroGene study. Eur Heart J 2006, 27:150-156

61. Communal C, Colucci WS, Remondino A, Sawyer DB, Port JD, Wichman SE, Bristow MR, Singh K: Reciprocal modulation of mitogen-activated protein kinases and mitogen-activated protein kinase phosphatase 1 and 2 in failing human myocardium. J Card Fail 2002, 8:86-92

62. Choudhary R, Palm-Leis A, Scott RC 3rd, Guleria RS, Rachut E, Baker KM, Pan J: All-trans retinoic acid prevents development of cardiac remodeling in aortic banded rats by inhibiting the reninangiotensin system. Am J Physiol Heart Circ Physiol 2008, 294: H633-H644

63. Ishikawa $\mathrm{Y}$, Komiyama K, Masuda S, Murakami M, Akasaka Y, Ito K, Akishima-Fukasawa Y, Kimura M, Fujimoto A, Kudo I, Ishii T: Expression of type $\mathrm{V}$ secretory phospholipase $\mathrm{A}(2)$ in myocardial remodelling after infarction. Histopathology 2005, 47:257-267 